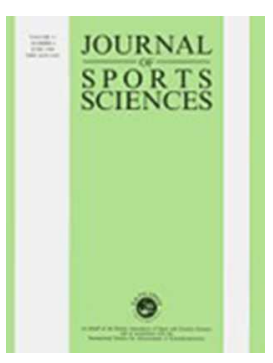

\title{
EFFECTS OF PSYCHOSOCIAL VARIABLES IN THE SIMILARITY AND INTERDEPENDENCE OF PHYSICAL ACTIVITY LEVELS AMONG ADOLESCENT BEST FRIEND DYADS
}

\begin{tabular}{|r|l|}
\hline Journal: & Journal of Sports Sciences \\
\hline Manuscript ID: & RJSP-2014-1196.R4 \\
\hline Manuscript Type: & Original Manuscript \\
\hline Keywords: & Best friends, Reciprocity, Dyad \\
\hline & \\
\end{tabular}

SCHOLARONE ${ }^{\mathrm{IM}}$

Manuscripts 


\title{
EFFECTS OF PSYCHOSOCIAL VARIABLES IN THE SIMILARITY AND INTERDEPENDENCE OF PHYSICAL ACTIVITY LEVELS AMONG ADOLESCENT BEST FRIEND DYADS
}

\begin{abstract}
Given that physical activity (PA) tends to decrease with age during adolescence, addressing factors that affect change are important. The present study examined the similarity and interdependence of PA as influenced by psychosocial factors among adolescent best friend dyads. Six hundred and sixty (660) adolescents, representing 330 best friend dyads, completed questionnaires with regard to PA, sitting time, perceived exercise benefits and barriers, physical self-perception and social support for PA. Dyads were also identified as reciprocal and non-reciprocal best friends; reciprocal means that both considered each other best friends and nonreciprocal were those in which only one considered the other a best friend. Data were analysed using a hierarchical linear model framework. Results indicated significant similarities between reciprocal best friend dyads for PA and sitting time, and for sitting time in non-reciprocal best friends ( $p s<.01)$. Psychosocial variables were associated with PA in reciprocal best friend dyads and with sitting time in reciprocal and non-reciprocal best friend dyads. Best friend gender, regular sports practice of the person, perceived exercise barriers of the best friend and best friend social support, were the best predictors for PA.
\end{abstract}




\section{Introduction}

The benefits of physical activity (PA) on health and wellbeing are extensively detailed in the literature (Janssen \& LeBlanc, 2010; Reiner, Niermann, Jekauc, \& Woll, 2013). According to the World Health Organization, physical inactivity is the fourth leading risk factor for death worldwide (WHO, 2014). In spite of the benefits, research indicates that most adolescents do not spend enough time in such endeavours (Hallal et al., 2012), and furthermore, PA levels tend to decrease with increasing age during adolescence (Lopes, Vasques, Maia, \& Ferreira, 2007; Nelson, Neumark-Stzainer, Hannan, Sirard, \& Story, 2006).

In order to address the problem of PA decline among adolescents, it is important to identify those factors that underscore and drive PA levels. From a proactive approach, knowledge of those factors could be used to create more effective interventions and health promotions strategies (Keresztes, Piko, Pluhar, \& Page, 2008; Salmon, Booth, Phongsavan, Murphy, \& Timperio, 2007; van Sluijs, McMinn, \& Griffin, 2007).

Physical activity is a complex multifactorial behaviour that is influenced by a variety of biological, behavioural and environmental factors and interactions. Two systematic reviews found that attitude, self-efficacy, goal orientation / motivation, physical education / school sports participation, family influences, and friend support were positively correlated with adolescent PA levels (Ferreira et al., 2007; Horst, Paw, Twisk, \& Mechelen, 
2007). Here, we report on an aspect of 'friend support' in the context of best friend dyads.

Underlying several theoretical views of friendship is the idea that adolescents have especially strong social identity needs. Needs that lead them to seek friends and spend increasing amounts of time that would typically be with family members [e.g., Fuligni (2001)]. Friends may influence an individual's motivation by enhancing competence, autonomy, and relatedness. Friends may also be a significant factor in building a sense of relatedness and autonomy to engage in independent PA (Russell Jago et al., 2009). This issue represents a relatively underexplored factor in the context of PA promotion. In general, the literature shows that peers and friends are important supporters of PA in both childhood and adolescence (Sharma et al., 2009). Social support is a key determinant of adolescent PA and friends support is often associated with higher PA in this age group (Hohepa, Scragg, Schofield, Kolt, \& Schaaf, 2007).

Significant others, including friends, in a young person's life is a factor that could contribute to a pleasant and effective PA experience. On the other hand, significant others may be a hindrance to a quality experience. The influence of peers and friends in adolescents' attitudes, behaviour and development in general and in PA in particular, are major potential factors (Smith, 2003). The present study differentiates the term 'friends' from peers with the idea that friendships represent a more significant relationship than peer associations. Furthermore, 'best friends' represent a higher level of relationship among a general set of friends. Despite the fact that adolescents 
could have several close friends, for the purposes of this study, we only considered best friend dyads.

Friends are typically similar on a wide range of characteristics such as gender, age, socioeconomic background, attitudes and interests (Bot, Engels, Knibbe, \& Meeus, 2005; Daddis, 2008; Kiesner, Poulin, \& Nicotra, 2003). Although social support from peers has been generally identified as a positive PA correlate (Duncan, Duncan, \& Strycker, 2005; Prochaska, Rodgers, \& Sallis, 2002); research about the role of friendship in PA adherence is limited. According to two comprehensive reviews, friends have an important role in PA behaviour of adolescents (Fitzgerald, Fitzgerald, \& Aherne, 2012; Maturo \& Cunningham, 2013). However, most investigations focused on friends in general and not specifically on close- or best friends. As opposed to friends in general, best friends are associated with higher levels of reciprocal influence and intimacy (Hays, 1984; Sharabany, 1994). More direct to our interests, research indicates that there is a mutually dependent relationship between adolescent friendship networks and PA. That is, adolescents tend to be friends with peers who engage in similar PA, and also emulate their friend's behaviours (de la Haye, Robins, Mohr, \& Wilson, 2011). Furthermore, reciprocal friends tend to be more similar in PA levels than nonreciprocal friends (Schofield, Mummery, Schofield, \& Hopkins, 2007). Reciprocal best friends are those in which both consider each other best friends, whereas non-reciprocal best friends are those in which only one friend considers the other his or her best friend. R. Jago, Macdonald-Wallis, et al. (2011) found that adolescent boys who have physically active best 
friends spend more minutes in moderate-to-vigorous PA, and girls who frequently take part in PA with their best friend achieve higher levels.

In a dyadic friend relationship a bidirectional influence is expected between the two persons involved, which could also result in an effect in PA behaviour of each person. Up to now, research has mostly focused on collecting individual data, assuming dyad effects by each person's perceptions about their friend's behaviour. In addition, studies have used more than one friend and not the best friend. Our position is that the effect of friendship on adolescent PA levels would be better understood by studying the influence of the individual's best friend. To date, very little is known about the effect of this dyadic relationship on PA patterns during adolescence.

Recently, Lopes, Gabbard, and Rodrigues (2013) found that dyads of best friends were similar in vigorous and moderate PA levels and sedentary behaviour. That study examined the influence of dyadic best friend relationship on PA, by collecting data from both friends and using the dyad as the unit of analysis in the context of the actor-partner interdependence model (Kenny, 1996). The actor-partner interdependence model is a model of dyadic relationships that integrates a conceptual view of interdependence in two-person relationships with the appropriate statistical techniques for measuring and testing it (Cook \& Kenny, 2005). This model addresses the concept of interdependence within an interacting dyad, based on the idea that an individual's behavior in a two person interaction is affected not only by the individual's own characteristics (actor effects), but also by the other person's characteristics (partner effects) and the individual's perceptions of that other person. Traditionally, actor effects have been estimated and 
partner effects often ignored. By studying only actor effects, researchers focus on the individual level of the analysis. However, by including partner effects, there is a possibility of identifying truly relational phenomena. In fact, the presence of partner effects implies that something relational has occurred in that a person's response depends upon some property of the partner (Kenny \& Cook, 1999).

A limitation of the Lopes et al. (2013) study was that it only included gender and age as predicting variables. With the present study, we used the same general methodology as Lopes et al. (2013). That is, we collected data on adolescent best friend's dyads and used the actor-partner interdependence model to analyse the dyadic interrelationships. The major differences were that we increased the number of potential predictors of the similarity and interdependence of PA levels, including a set of psychosocial and demographic variables, namely: perceived exercise benefits and barriers, physical self-perception, perception of social support from significant others.

\section{Methods}

\section{Participants and design}

Data were collected during May 2012 and participants were adolescents of both sexes, recruited from two secondary schools in Bragança, a county in the northeast of Portugal. All students were invited to participate with refusal minimal $(0.01 \%)$, resulting in a sample of 660 adolescents aged 12 - to 20 years (males $n=303$, girls $n=357$ ) with a mean of $15.8 \pm 1.9$. Permission to interview the participants was obtained from school directors and written 
informed consent was obtained from the students and their parents. The study was approved by the ethics committee of UTAD, Vila Real.

All questionnaires were completed in class during regular school hours. Each class had about 25 students and they took approximately 20 minutes to complete all questionnaires. The same two examiners (trained and experienced) collected all data; they explained the purpose of each questionnaire and clarified any doubts raised by participants. To determine test-retest reliability, a randomized subsample of 30 participants was measured one week apart.

To determine friendship dyads, definitions of various forms of friendship were presented to participants. They were then asked to complete a friendship questionnaire indicating the number of close friends and nominating their one best friend who was not a brother or sister. Friends were identified in two categories. A 'best friend' was described as "the one you spend most of your time with and can tell everything to and is always there for you." A 'close friend' was "someone that is there for you and that you get along with, but you don't spend a lot of time with." Friend type was identified with an alphanumeric code. These alphanumeric codes were entered in a database along with all participants' data and used to pair the best friend dyads. As noted earlier, reciprocal best friends are those in which both consider each other best friends, whereas non-reciprocal best friends are those in which only one friend considers the other his or her best friend.

This process resulted in 330 best friendship dyads. Of these dyads, 167 were reciprocal best friends and 163 non-reciprocal best friends; 277 dyads were of the same sex (girls $n=152$ dyads, boys $n=125$ dyads), and 53 
dyads were of opposite sex. All participants were white European, and the majority $(75 \%)$ were classified in same socioeconomic group, level 1 (the highest level) and 25\% were classified in level 2 (Graffar, 1956).

\section{Measures and procedure}

Physical activity and sedentary behaviour. Physical activity behaviour was determined with the short version of the International Physical Activity Questionnaire (Hagströmer, Ekelund, Yngve, \& Sjöström, 2002). The questionnaire was self-administrated with reference to the last 7 days of recalled PA. The International Physical Activity Questionnaire short form asks about three specific types of activity carried out in three domains: leisure time, domestic and gardening / yard activities; work-related activities (in the context of the present study this domain was switched to school-related physical activity, including activity during physical education classes and breaks); and, transport-related activity. The specific types of activity assessed were walking, moderate-intensity activities and vigorous intensity activities. Frequency (measured in days per week) and duration (time per day) were collected separately for each specific type of activity. The items were structured to provide separate scores on walking; moderate PA; and vigorous PA, as well as a combined total score to describe overall level of activity, expressed in metabolic equivalents per minutes per week (MET I minutes / week), using the following formulas:

\footnotetext{
Walking: MET-minutes / week $=3.3$ * walking minutes * walking 'days'

Moderate: MET-minutes/week $=4.0 *$ moderate-intensity activity minutes* moderate days
} 
Vigorous: MET-minutes / week $=8.0$ * vigorous-intensity activity minutes* vigorous-intensity days

A combined total physical activity MET - min / week was computed as the sum of Walking + Moderate + Vigorous MET - min / week. More details about International Physical Activity Questionnaire can be found at the website: https://sites.google.com/site/theipaq.

Also included in International Physical Activity Questionnaire is a sitting question that is a supplemental indicator variable of sedentary behaviour which was not included in the summary score of PA; measured in hours per day. The short version of the International Physical Activity Questionnaire has been tested extensively with reported reliability of 0.80 and validity of 0.30 (Craig et al., 2003). In Portuguese speaking adolescents, the reported reliability is 0.49 to 0.83 and validity 0.24 to 0.55 (Guedes, Lopes, \& Guedes, 2005). The Bland-Altman (Bland \& Altman, 1986) analysis for test re-test reliability of the International Physical Activity Questionnaire for the present study for the combined total physical activity index indicates that the $95 \%$ limits of agreement between the two measures ranged from 0.23 to 1.94 , with an agreement ratio (Nevill \& Atkinson, 1997) of $0.85(0.55)$.

In addition to the International Physical Activity Questionnaire, participants in the present study were asked if they participate in formal sports and if so, how many times per week.

\section{Psychosocial variables.}

Perceived exercise benefits and barriers were assessed by the Exercise Benefits / Barriers Scale Questionnaire (Sechrist, Walker, \& Pender, 
1987) comprised of two components: benefits and barriers. The benefit component consists of 29 items and the barrier component 14 items. All items of both scales are scored on a Likert 4-point response format, where 1 = 'strongly disagree'; 2 = 'disagree'; 3 = 'agree'; and 4 = 'strongly agree'. In the present study the two components were determined separated. The benefits scale varies between 29 and 116 points and the barriers scale between 14 and 56 points. The reported internal consistency (Cronbach's alpha) for the benefits and the barriers scales are 0.95 and 0.86 respectively, while test re-test reliability is 0.89 and 0.77 respectively (Gyurcsik, Spink, Bray, Chad, \& Kwan, 2006). The internal consistencies (Cronbach's alpha) of the benefits and barriers scale for our sample were 0.87 and 0.93 respectively. The Bland-Altman (Bland \& Altman, 1986) analysis for test retest reliability for the barriers scale indicates that the $95 \%$ limits of agreement between the two measures ranged from 0.48 to 1.96 , with an agreement ratio of 1.08(0.38); for the benefits scale the limits of agreement ranged from 0.88 to 1.14, with an agreement ration (Nevill \& Atkinson, 1997) of 1.01(0.07).

Physical self-perception was assessed with the Portuguese version (Bernardo \& Matos, 2003) of the Physical Self-Perception Profile for Children and Youth (Bernardo \& Matos, 2003) and with the Perceived Importance Profile for Children and Youth (Whitehead, 1995). The Physical SelfPerception Profile for Children and Youth consists of 36 items and uses Harter's (1982) structured alternative format designed to minimize the tendency towards socially desirable responses. This instrument has six subscales: sport competence, physical condition, attractive body, physical strength, physical self-worth and global self-esteem. Each subscale consists 
of 6 items in which participants are presented with two contrasting descriptions (e.g., those with unattractive bodies and those with attractive bodies) and are asked which description is most like themselves and whether the description they select is "sort of true" or "really true" for them. Item scores can range from 1 to 4 . A value of 3 or 4 represents a positive perception and value of 2 or 1 a negative perception. The result of each subscale is obtained with the average of 6 items belonging to the scale. The reported internal consistency (Cronbach's alpha) for the different subscales were between 0.73 and 0.85 , while test re-test reliability were between 0.71 and 0.77 (Bernardo \& Matos, 2003). Cronbach's alpha values for our sample varied between 0.51 and 0.89 .

The Bland-Altman (Bland \& Altman, 1986) analysis for test re-test reliability for the different subscales indicates that the lower limit of $95 \%$ limits of agreement between the two measures varied between 0.54 and 0.66 , and the upper limit varied between 1.43 and 1.47 , with an agreement ratio (Nevill \& Atkinson, 1997) varying between $0.99(0.23)$ and $1.07(0.21)$.

The Perceived Importance Profile for Children and Youth contains four subscales (attractive body importance, sport / athletic competence importance, strength competence importance, and physical condition competence importance) with two items each. The instrument also uses Harter's (1982) structured alternative format. For the present study, Cronbach's alpha varied between 0.51 and 0.62. The Bland-Altman (Bland \& Altman, 1986) analysis for test re-test reliability for the different subscales indicates that the lower limit of $95 \%$ limits of agreement between the two measures varied between 0.28 and 0.55 , and the upper limit varied between 
1.45 and 1.51, with an agreement ratio (Nevill \& Atkinson, 1997) varying between $1.0(0.23)$ and 1.04(0.38).

Perception of social support of best friend for PA was assessed with an adaptation for Portuguese language of the Friend Support Scale (R. Jago, Page, \& Cooper, 2012), which is an adaptation of Prochaska et al. (2002) Peers Support Scale. The perception of support for physical activity was evaluated based on responses to the following four questions prefaced with "how often do your best friend:

1) Encourage you to exercise or play sports,

2) Exercise or play sports with you,

3) Tell you that you are doing well in exercise or sports,

4) Watch you take part in exercise or sports?"

All items were assessed on a 4-point scale (1 - never to 4 - very often). Each participant had to answer all items, otherwise his / her data were excluded from the analysis. The four responses were averaged to produce the scale score. Internal consistencies (Cronbach's alpha) was 0.87 . The Bland-Altman (Bland \& Altman, 1986) analysis for test re-test reliability indicates that the $95 \%$ limits of agreement between the two measures ranged from 0.64 to 1.47 , with an agreement ratio (Nevill \& Atkinson, 1997) of 1.05(0.21)

\section{Data analysis}

Descriptive statistics (means and standard deviations) were calculated for all variables. 
The interdependence of PA levels between best friend dyads was analysed in the context of the actor-partner interdependence model (Kenny, 1996). The actor-partner interdependence model is a data analytic technique designed for non-independent data, which simultaneously estimates the effect that an individual's predictor variables have on his or her own outcome variables and on his or her partner's outcome variables. The model has the potential of including several variables from both persons in the dyad, including those that research has shown to correlate with PA (Pedro Silva, 2014; Sallis, Prochaska, \& Taylor, 2000), therefore bringing some insight to the effect interpersonal relationships have on adolescent PA levels. With that model, the independent variables (sex, age, and psychosocial characteristics of each of the two dyad friends), designated as $X$ and $X '$, were predictors of their own (actor) PA level and of their best friend (partner) PA (designated as $Y$ and $\left.Y^{\prime}\right)$. The model postulates that in each dyad, independent variables are correlated (as indicated by the two-headed arrow between $\mathrm{X}$ and $\mathrm{X}^{\prime}$ ); and the unexplained variance for individual PA levels (represented by $U$ and $U$ ') are correlated. This correlation represented the partial correlation between PA level of both individuals in the dyad ( $Y$ and $\left.Y^{\prime}\right)$ controlling for the independent variables of both individuals in the dyad ( $X$ and $\left.X^{\prime}\right)$. The actor-partner interdependence model hypothesizes that an individual's predictor variable will influence not only her / his own PA level (the actor effect), but also the partner's PA level (the partner effect) (Figure 1).

The actor-partner interdependence model was tested via a hierarchical linear model, independently for reciprocal and non-reciprocal best friend dyads. First, a null model (without predictors) was run to calculate the 
similarity of PA behaviours (vigorous PA, moderate PA, walking, and sitting time) between all friend dyads using ICC. When a similarity was found in each outcome (vigorous PA, moderate PA, walking, and sitting time); that is, a significant ICC, a new model was run with psychosocial variables. Predictor variables were excluded from the model if they had no significant effect; the final model for each outcome only contained significant predictors.

The hierarchical linear model was used to take advantage of the complex hierarchical nature of the dyadic data by separating the variance of the scores into two levels of analysis: individual and the dyad. The data is said to be hierarchically organized, given that individuals are nested within their respective dyad. In order to make the results more interpretable, predictor variables were centered around the mean.

All statistical test were considered significant with $p \leq 0.05$

Figure 1 near here

\section{Results}

Descriptive statistics for reciprocal and non-reciprocal best friend dyads and the entire sample are summarized in Table 1. Levels of PA were quite similar between reciprocal and non-reciprocal best friends' dyads. The only significant differences were in overall level of activity and moderate PA. For psychosocial variables, there were no significant differences between dyads. The means for perceived exercise benefits for both reciprocal and nonreciprocal dyads and for the entire sample were above the mid-point of the 
scale (72.5), which means that adolescents perceived high benefits of PA. The mean values for perceived exercise barriers were also above the midpoint of the scale (35), suggesting that adolescents perceived low PA barriers.

All subscales values for the Physical Self-Perception Profile for Children and Youth were positive (above 2.5, the mid-point of the scale) for both reciprocal and non-reciprocal dyads and for the entire sample. Results of the Perceived Importance Profile, except for the 'attractive body importance' subscale, were slightly below the mid-point of the scale for both dyads and for the sample as a whole. Reciprocal and non-reciprocal samples had high perception values for PA support from friends and best friends. For parents, the value was slightly lower, but still in the positive direction.

\section{Table 1 near here}

\section{Table 2 near here}

Friendship reciprocity was first tested as predictor in all dyads, but was not significant. Based on that outcome, we conducted separate analysis for the different types of friendship. That option allowed us to further explore the effects separately for reciprocal and non-reciprocal dyads.

Results from the null models on reciprocal dyads indicated a significant ICC of $0.23(p=0.002)$ for overall PA and $0.39(p<0.001)$ for sitting time. For vigorous PA, moderate PA and Walking, ICC values were not significant. For non-reciprocal dyads, ICC were only significant for sitting time [ICC = 
$0.21(p=0.008)]$. These results showed moderate but significant similarity in overall PA and sitting time between reciprocal best friends and for sitting time in non-reciprocal best friend dyads.

Looking for possible factors that could explain the interdependence of PA levels within best friend dyads, predictors were included (psychosocial variables, sex and age) in the hierarchical linear model for explaining similarity on overall PA and sitting time in reciprocal and non-reciprocal best friend dyads. The results for significant predictors, that is, the final models, are shown in Tables 2 and 3. For all models, the intercept value represents the estimation of average for dyads, since the dependent variables were centered around the mean.

\section{Table 2 near here}

\section{Table 3 near here}

In best friend reciprocal dyads, the significant predictors for overall PA were the sex of the partner, participation of the partner in formal sports, perception of the partner barriers for PA, sport competence importance of the partner, and perception of the partner of best friend social support. This suggests that having a male as best friend results in extra PA by 1025.3 MET / minutes / week, compared to having a female best friend. If the subject participated in formal sports the estimation of overall mean PA increased 955.6 MET / minutes / week, compared to if the individual did not participate. 
The perceived barriers for PA of the best friend were an impediment for overall PA. For each unit increase in perceived barriers of the best friend, the estimation of overall mean PA of the subject decreased 85.4 MET / minutes / week. For each unit increase in the importance attributed to sport competence by the best friend, overall mean PA estimation increased 686.5 MET / minutes / week. For each unit of the perception of the partner of best friend social support, overall mean PA estimation increased 213.9 MET / minutes / week.

Only the Physical Self-Perception Profile for Children and Youth factor of 'sport competence of the partner' was found to be a predictor of sitting time in reciprocal best friend dyads. For each unit change in this variable, mean sitting time of the reciprocal best friends decreased $0.61 \mathrm{~h} /$ day.

In non-reciprocal best friend dyads the significant predictors for sitting time were the sex of the individual, sport competence importance of the partner, and physical condition competence importance. Which means that, if the individual was a male, mean sitting time estimation was $0.7 \mathrm{~h} /$ day higher than if it was a female. However, this factor decreased $0.4 \mathrm{~h} /$ day for each unit increase in the importance attributed to sport competence by the best friend, and $0.4 \mathrm{~h} /$ day for each unit increase in the importance attributed to physical condition competence importance by the best friend.

\section{Discussion}

This study examined factors associated with similarity and interdependence of PA levels among adolescent best friend dyads. We assumed that the interdependence could be explained by a set of 
psychosocial and demographic variables. In the context of PA promotion in adolescents, this issue is still relatively unexplored.

We found that reciprocal best friend dyads were significantly similar in overall PA and sitting time, and non-reciprocal best friend dyads were only similar in sitting time. These results are quite analogous to those previously reported. For example, Anderssen and Wold (1992), although they used a broader scope of "best friends" (peers, friends, and best friends), found a moderate correlation between adolescent boys $(r=0.23)$ and girls $(r=0.31)$ and best friends' PA. R. Jago, Macdonald-Wallis, et al. (2011) reported that moderate PA was associated with frequency of activity of girl best friends (ages 10- to 11 years), and that for boys (same ages) moderate PA was associated with their best friend's moderate PA. More recently, Lopes et al. (2013) found that best friend dyads, independent of being reciprocal or nonreciprocal best friends, were similar on moderate $(\mathrm{ICC}=0.31)$ and vigorous $(I C C=0.32) P A$, and on sitting time $(I C C=0.21)$.

We wish to note that in the present study, non-reciprocal best friend dyads were only similar for sitting time, unlike previous studies that suggested reciprocity was not a predictor of best friend dyad similarity (Lopes et al., 2013). The difference between the results of the two studies could be due to the sample. With the present study, the sample was much larger than that with Lopes et al. (2013). In addition, the region of data collection was different, prompting us to speculate that perhaps culture and socioeconomic characteristics were different. Possible reasons for the differences between reciprocal and no-reciprocal best friends may be explained by the possibility 
that non-reciprocal best friends do not influence or support each other as much as reciprocal best friends.

Similarities in PA may not always be a characteristic of friendship, and it is not clear whether similarities or differences arise because of a selection process (youth form relationships with peers who have similar behaviours) or because of an influence process (youth change their behaviours to emulate their friends) (de la Haye et al., 2011). Furthermore, the resemblance between friends' PA could depend on replicating behaviour of the friend, or on friends' social support and encouragement. R. Jago, Macdonald-Wallis, et al. (2011) suggested that among best friends, modelling and spending time being active together are important ways that friendship influence is demonstrated and continually reinforced. Modelling and co-participation could be the factors that lead best friends to be similar in PA. Therefore, psychosocial and demographic variables such as friends support for PA, sex of the best friend, participation in organized sports, physical self-perception, perceived exercise benefits and barriers, may also have an influence in the similarity between friends' PA levels.

In our study, of all the variables tested to explain the interdependence of PA behaviour within best friend dyads, the best predictors for reciprocal best friend's overall PA were the "sex of the partner", "participation of the actor in formal sports", "perceived barriers for PA of the partner" (inverse relationship), "sport competence importance of the partner", and "perception of the partner of best friend social support". It is important to note that all of these variables, with exception of "participation in formal sports", were characteristics of the best friend (partner).

It would appear that, independent of the sex of the individual, having a male best friend is a factor in being more active. It is well described in the literature that male children and adolescents are more active than females 
(Belcher et al., 2010; Klasson-Heggebo \& Anderssen, 2003; Lopes et al., 2013). Our results are similar to those found by Lopes et al. (2013), which confirm that having a male best friend, regardless of the sex of the other partner, could act as a positive factor to enhance PA in the best friend dyad. The importance ascribed to sport competence by the best friend and the perception of the social support from the other element of the dyad are positive factors for PA. But, as expected, when the best friend perceives barriers for PA, it results in a negative factor for his / her dyad peer PA. These results are evidence of the importance of best friend characteristics to the interdependence of PA within best friend dyads.

The only predictor for sitting time in reciprocal best friend dyads was "sport competence of the partner", and for non-reciprocal best friend dyads "sex of the actor", "sport competence importance of the partner", and "physical condition competence importance of the actor". From our perspective, the differences between dyads reinforces the importance of the 'reciprocal' best friend relationship associated with sitting time behaviour. In fact, data shows that when a reciprocal best friend steps into the relationship, the actor's own characteristics (sex, and self-physical condition competence) are no longer significant for determining sitting time behaviour. That is, in a reciprocal best friend dyad, the partner's sport competence turns out to be the most important factor in sitting behaviour, supplanting even the actor's characteristics.

In general, the findings of the present study indicate that best friends have similar PA levels and some characteristics of the best friend seems to be factors that contribute to increased PA level; namely, the sex (male), perceived barriers for PA (inverse relationship), sport competence importance and perception of best friend social support. PA intervention programs tend to ignore the extent to which an adolescent friend may affect 
adolescent PA (Demetriou \& Höner, 2012; R. Jago, Page, \& Cooper, 2011). Strategies to foster friend support for PA may be important for helping adolescents to be more physically active. We advocate that PA intervention programs for adolescents should have a social approach. That is, focus on increasing PA by structuring the social environment so that it includes significant others, especially best friends.

In regard to limitations of this work, the cross-sectional design does not allow inference of cause and effect. We believe that a longitudinal design will better elucidate the mechanisms through which friendships during adolescence influence PA. Another limitation is that PA was assessed via questionnaire. Despite the fact that the test retest reliability of the questionnaire was good (ICC $=0.59$ to 0.75 ), self-reported PA assessment has a potential bias problem of subjectivity and the necessity of recall, leading to possible inaccuracy of the measurements. However, questionnaires were the best choice to evaluate PA in a relatively short period, given the sample size.

\section{Conclusions}

Results indicate that reciprocal best friend dyads were similar in PA and sitting time, and non-reciprocal dyads were similar on sitting time. Furthermore, we found that specific psychosocial factors were associated with PA and sitting time. The sex of best friends, regular sports participation, perceived exercise barriers of the best friend and best friend social support, were the best predictors for PA. Overall, this work reinforces the idea that specific characteristics and behaviours of significant others, namely best friends as shown here, are associated with adolescent physical activity. 


\section{References}

Anderssen, N., \& Wold, B. (1992). Parental and peer influences on leisuretime physical activity in young adolescents. Research Quarterly for Exercise and Sport, 63(4), 341-348.

Belcher, B. R., Berrigan, D., Dodd, K. W., Emken, B. A., Chou, C.-P., \& Spruijt-Metz, D. (2010). Physical Activity in US Youth: Effect of Race/Ethnicity, Age, Gender, and Weight Status. Medicine \& Science in Sports \& Exercise, 42(12), 2211-2221. doi:

10.1249/MSS.0b013e3181e1fba9

Bernardo, R. P. S., \& Matos, M. G. (2003). Adaptação portuguesa do physical self-perception profile for children and youth e do perceived importance profile for children and youth. Análise Psicológica, 2(XX1), 127-144.

Bland, J. M., \& Altman, D. G. (1986). Statistical methods for assessing agreement between two methods of clinical measurement. Lancet, $i$, 307-310.

Bot, S. M., Engels, R. C. M. E., Knibbe, R. A., \& Meeus, W. H. J. (2005). Friend's drinking behaviour and adolescent alcohol consumption: The moderating role of friendship characteristics. Addictive Behaviors, 30(5), 929-947.

Cook, W. L., \& Kenny, D. A. (2005). The Actor-Partner Interdependence Model: A model of bidirectional effects in developmental studies. International Journal of Behavioral Development, 29(2), 101-109. doi: 10.1080/01650250444000405

Craig, C. L., Marshall, A. L., Sjöström, M., Bauman, A. E., Booth, M. L., Ainsworth, B. E., ... Oja, P. (2003). International Physical Activity Questionnaire: 12-Country Reliability and Validity. Medicine and Science in Sports and Exercise, 35(8), 1381.

Daddis, C. (2008). Similarity between Early and Middle Adolescent Close Friends' Beliefs about Personal Jurisdiction. Social Development, 17(4), 1019-1038. doi: 10.1111/j.1467-9507.2008.00471.x

de la Haye, K., Robins, G., Mohr, P., \& Wilson, C. (2011). How physical activity shapes, and is shaped by, adolescent friendships. Social Science \& Medicine, 73(5), 719-728. doi: http://dx.doi.org/10.1016/j.socscimed.2011.06.023

Demetriou, Y., \& Höner, O. (2012). Physical activity interventions in the school setting: A systematic review. Psychology of Sport and Exercise, 13(2), 186-196. doi: http://dx.doi.org/10.1016/j.psychsport.2011.11.006

Duncan, S. C., Duncan, T. E., \& Strycker, L. A. (2005). Sources and Types of Social Support in Youth Physical Activity. Health Psychology, 24(1), 310. doi: $10.1037 / 0278-6133.24 .1 .3$ 
Ferreira, I., Van Der Horst, K., Wendel-Vos, W., Kremers, S., Van Lenthe, F. J., \& Brug, J. (2007). Environmental correlates of physical activity in youth - a review and update. Obesity Reviews, 8(2), 129-154. doi: 10.1111/j.1467-789X.2006.00264.x

Fitzgerald, A., Fitzgerald, N., \& Aherne, C. (2012). Do peers matter? A review of peer and/or friends' influence on physical activity among American adolescents. Journal of Adolescence, 35(4), 941-958. doi: http://dx.doi.org/10.1016/j.adolescence.2012.01.002

Fuligni, A. J. E., Jacquelynne S.; Barber, Bonnie L.; Clements, Peggy. (2001). Early adolescent peer orientation and adjustment during high school. Developmental Psychology, 37(1), 28-36. doi: 10.1037/00121649.37.1.28

Graffar, M. (1956). Une méthode de classification sociale d'échantillons de population. Courrier, 6(8), 455-459.

Guedes, D. P., Lopes, C. C., \& Guedes, J. E. R. P. (2005). Reprodutibilidade e validade do Questionário Internacional de Atividade Física em adolescentes. Revista Brasileira de Medicina do Esporte, 11, 151-158.

Gyurcsik, N. C., Spink, K. S., Bray, S. R., Chad, K., \& Kwan, M. (2006). An ecologically based examination of barriers to physical activity in students from grade seven through first-year university. The Journal of adolescent health : official publication of the Society for Adolescent Medicine, 38(6), 704-711.

Hagströmer, M., Ekelund, U., Yngve, A., \& Sjöström, M. (2002). A validity study of ipaq versus two indirect and two direct measures of physical activity. Medicine and Science in Sports and Exercise, 34(5 Supplement 1), S139.

Hallal, P. C., Andersen, L. B., Bull, F. C., Guthold, R., Haskell, W., \& Ekelund, U. (2012). Global physical activity levels: surveillance progress, pitfalls, and prospects. The Lancet, 380(9838), 247-257.

Harter, S. (1982). The Perceived Competence Scale for Children. Child Development, 53, 87-97.

Hays, R. B. (1984). The Development and Maintenance of Friendship. Journal of Social and Personal Relationships, 1(1), 75-98. doi: 10.1177/0265407584011005

Hohepa, M., Scragg, R., Schofield, G., Kolt, G., \& Schaaf, D. (2007). Social support for youth physical activity: Importance of siblings, parents, friends and school support across a segmented school day. International Journal of Behavioral Nutrition and Physical Activity, 4(1), 54.

Horst, K. V. D., Paw, M. J. C. A., Twisk, J. W. R., \& Mechelen, W. V. (2007). A Brief Review on Correlates of Physical Activity and Sedentariness in Youth. Medicine and Science in Sports and Exercise, 39(8), 12411250.

Jago, R., Brockman, R., Fox, K., Cartwright, K., Page, A., \& Thompson, J. (2009). Friendship groups and physical activity: qualitative findings on 
how physical activity is initiated and maintained among 10-11 year old children. International Journal of Behavioral Nutrition and Physical Activity, 6(1), 4.

Jago, R., Macdonald-Wallis, K., Thompson, J. L., Page, A. S., Brockman, R., \& Fox, K. R. (2011). Better with a buddy: influence of best friends on children's physical activity. Medicine \& Science in Sports \& Exercise, 43(2), 259-265. doi: 10.1249/MSS.0b013e3181edefaa

Jago, R., Page, A. S., \& Cooper, A. R. (2011). Friends and Physical Activity during the Transition from Primary to Secondary School. Medicine \& Science in Sports \& Exercise. doi: 10.1249/MSS.0b013e318229df6e

Jago, R., Page, A. S., \& Cooper, A. R. (2012). Friends and Physical Activity during the Transition from Primary to Secondary School. Medicine and Science in Sports and Exercise, 44(1), 111-117. doi: Doi 10.1249/Mss.0b013e318229df6e

Janssen, I., \& LeBlanc, A. (2010). Systematic review of the health benefits of physical activity and fitness in school-aged children and youth. International Journal of Behavioral Nutrition and Physical Activity, 7(1), 40.

Kenny, D. A. (1996). Models of Non-Independence in Dyadic Research. Journal of Social and Personal Relationships, 13(2), 279-294. doi: 10.1177/0265407596132007

Kenny, D. A., \& Cook, W. (1999). Partner effects in relationship research: conceptual issues, analytic difficulties, and ilustrations. Personal Relationship, 6, 433-448.

Keresztes, N., Piko, B. F., Pluhar, Z. F., \& Page, R. M. (2008). Social influences in sports activity among adolescents. The Journal of the Royal Society for the Promotion of Health, 128(1), 21-25.

Kiesner, J., Poulin, F., \& Nicotra, E. (2003). Peer Relations Across Contexts: Individual-Network Homophily and Network Inclusion In and After School. Child Development, 74(5), 1328-1343. doi: 10.1111/14678624.00610

Klasson-Heggebo, L., \& Anderssen, S. A. (2003). Gender and age differences in relation to the recommendations of physical activity among Norwegian children and youth. Scandinavian Journal of Medicine \& Science in Sports, 13(5), 293-298.

Lopes, V. P., Gabbard, C., \& Rodrigues, L. P. (2013). Physical Activity in Adolescents: Examining Influence of the Best Friend Dyad. Journal of Adolescent Health, 52(6), 752-756. doi: http://dx.doi.org/10.1016/j.jadohealth.2012.12.004

Lopes, V. P., Vasques, C. M. S., Maia, J. A. R., \& Ferreira, J. C. V. (2007). Habitual physical activity levels in childhood and adolescence assessed with accelerometry. Journal of Sports Medicine and Physical Fitness, 47(2), 217-222. 
Maturo, C. C., \& Cunningham, S. A. (2013). Influence of Friends on Children's Physical Activity: A Review. American Journal of Public Health, 103(7), e23-e38. doi: 10.2105/AJPH.2013.301366

Nelson, M. C., Neumark-Stzainer, D., Hannan, P. J., Sirard, J. R., \& Story, M. (2006). Longitudinal and Secular Trends in Physical Activity and Sedentary Behavior During Adolescence. Pediatrics, 118(6), e16271634. doi: 10.1542/peds.2006-0926

Nevill, A. M., \& Atkinson, G. (1997). Assessing agreement between measurements recorded on a ratio scale in sports medicine and sports science. British Journal of Sports Medicine, 31(4), 314-318.

Pedro Silva, R. L., Jorge Mota, Greg Welk. (2014). Direct and Indirect Effects of Social Support on Youth Physical Activity Behavior. Pediatric Exercise Science, 26(1), 86 - 94.

Prochaska, J. J., Rodgers, M. W., \& Sallis, J. F. (2002). Association of Parent and Peer Support with Adolescent Physical Activity. Research Quarterly for Exercise and Sport, 73(2), 206-210.

Reiner, M., Niermann, C., Jekauc, D., \& Woll, A. (2013). Long-term health benefits of physical activity - a systematic review of longitudinal studies. BMC Public Health, 13(1), 813.

Sallis, J. F., Prochaska, J. J., \& Taylor, W. C. (2000). A review of correlates of physical activity of children and adolescents. Medicine and Science in Sports and Exercise, 32(5), 963-975.

Salmon, J., Booth, M. L., Phongsavan, P., Murphy, N., \& Timperio, A. (2007). Promoting Physical Activity Participation among Children and Adolescents. Epidemiologic Reviews, 29(1), 144-159. doi: 10.1093/epirev/mxm010

Schofield, L., Mummery, W. K., Schofield, G., \& Hopkins, W. (2007). The association of objectively determined physical activity behavior among adolescent female friends. Research Quarterly for Exercise and Sport, 78(2), 9-15.

Sechrist, K. R., Walker, S. N., \& Pender, N. J. (1987). Development and psychometric evaluation of the exercise benefits/barriers scale. Research in Nursing and Health, 10, 357-365.

Sharabany, R. (1994). Intimate Friendship Scale: Conceptual Underpinnings, Psychometric Properties and Construct Validity. Journal of Social and Personal Relationships, 11(3), 449-469. doi: 10.1177/0265407594113010

Sharma, S. V., Hoelscher, D. M., Kelder, S. H., Diamond, P. M., Day, R. S., \& Hergenroeder, A. C. (2009). A Path Analysis to Identify the Psychosocial Factors Influencing Physical Activity and Bone Health in Middle-School Girls. Journal of Physical Activity and Health, 6(5), 606616.

Smith, A. L. (2003). Peer relationships in physical activity contexts: a road less traveled in youth sport and exercise psychology research. 
Psychology of Sport and Exercise, 4(1), 25-39. doi: 10.1016/s14690292(02)00015-8

van Sluijs, E. M. F., McMinn, A. M., \& Griffin, S. J. (2007). Effectiveness of interventions to promote physical activity in children and adolescents: systematic review of controlled trials. The BMJ, 335, 703. doi: 10.1136/bmj.39320.843947.BE

Whitehead, J. R. (1995). A Study of Children's Physical Self-Perceptions Using an Adapted Physical Self-Perception Profile Questionnaire. Pediatric Exercise Science, 7(2), 132-151.

WHO. (2014). Physical activity. Fact sheet №385. Retrieved 15-04-2014, 2014, from http://www.who.int/mediacentre/factsheets/fs385/en/ 
Figure 1: Actor-partner interdependence model. Horizontal lines are actor effects (a) and diagonal lines are partner effects ( $p)$. Adapted from Kenny (1996). 
Table 1: Descriptive statistics (mean and standard-deviation) for whole sample and split in reciprocal and no-reciprocal best friends.

\begin{tabular}{|c|c|c|c|c|}
\hline & Whole sample & Reciprocal & No-reciprocal & \\
\hline $\mathrm{N}$ & 660 & 326 & 334 & \\
\hline Age & $15.8(1.9)$ & $15.8(1.9)$ & $15.8(1.8)$ & \\
\hline Sport participation (\% of yes) & 71.3 & 67.0 & 75.6 & \\
\hline GPA (MET/minutes/week) & 2977 (2493) & $2791(2418)$ & $3172(2560)$ & * \\
\hline VPA (MET/minutes/week) & $2015(1746)$ & $1949(1833)$ & $2080(1666)$ & \\
\hline MPA (MET/minutes/week) & $802(700)$ & $453(603)$ & $673(821)$ & * \\
\hline Walking (MET/minutes/week) & $845(1042)$ & $874(1017)$ & $818(1070)$ & \\
\hline Sitting time (h/day) & $10(2)$ & $10(2)$ & $10(2)$ & \\
\hline \multicolumn{5}{|l|}{ Perceived exercise benefits and barriers } \\
\hline Benefits & $94.8(10.9)$ & $94.6(10.4)$ & $95.1(11.4)$ & \\
\hline Barriers & $41.3(6.6)$ & $41.7(5.9)$ & $40.9(7.2)$ & \\
\hline \multicolumn{5}{|c|}{ Physical Self-Perception Profile for Children and Youth } \\
\hline Global self-esteem & $3.1(0.6)$ & $3.1(0.6)$ & $3.2(0.6)$ & \\
\hline Physical self-worth & $2.8(0.7)$ & $2.8(0.7)$ & $2.9(0.7)$ & \\
\hline Sport competence & $2.8(0.6)$ & $2.8(0.6)$ & $2.8(0.5)$ & \\
\hline Physical condition & $2.9(0.6)$ & $2.8(0.6)$ & $2.8(0.6)$ & \\
\hline Attractive body & $2.6(0.7)$ & $2.6(0.7)$ & $2.7(0.6)$ & \\
\hline Physical strength & $2.5(0.6)$ & $2.5(0.6)$ & $2.6(0.6$ & \\
\hline \multicolumn{5}{|c|}{ Perceived Importance Profile for Children and Youth } \\
\hline Sport competence importance & $2.4(0.7)$ & $2.4(0.6)$ & $2.4(0.7)$ & \\
\hline Physical condition competence importance & $2.4(0.7)$ & $2.4(0.6)$ & $2.3(0.7)$ & \\
\hline Attractive body importance & $2.6(0.7)$ & $2.6(0.6)$ & $2.6(0.7)$ & \\
\hline Strength competence importance & $2.4(0.6)$ & $2.5(0.6)$ & $2.4(0.6)$ & \\
\hline \multicolumn{5}{|l|}{ Perception of social support of significant others } \\
\hline Friends support & $3.2(0.6)$ & $3.2(0.6)$ & $3.2(0.6)$ & \\
\hline
\end{tabular}


Best friend support

$3.1(0.7)$

$3.1(0.7)$

$3.1(0.7)$

Parents support

$2.8(0.7)$

$2.8(0.7)$

$2.8(0.7)$

GPA: overall level of physical activity, VPA: vigorous physical activity; MPA: moderate physical activity, SB: sedentary behavior; MET: metabolic equivalent. * Significant difference for $p<0.05$ between reciprocal and no-reciprocal best friends' dyads. 
Table 2: Specification of parameters (fixed effects) in final models [overall physical activity (MET/minutes/week) and sitting time (h/day) as dependent variables] for reciprocal best friend dyads with predictors, with standard errors (SE), confidence intervals $(\mathrm{Cl})$, and effects size (ES).

\begin{tabular}{|c|c|c|c|}
\hline Parameter & Estimate (SE) & $95 \% \mathrm{Cl}$ & ES \\
\hline \multicolumn{4}{|l|}{ Overall PA } \\
\hline Intercept & $1797.3(260.6)$ & $1283.8-2310.9$ & \\
\hline Sex of the partner (male $=1 ;$ female $=0$ ) & $1025.3(298.5)$ & $436.5-1614.2$ & 0.14 \\
\hline Participation of the actor in formal sport (yes $=1 ;$ no $=0$ ) & $955.6(301.1)$ & $362.8-1548.5$ & 0.12 \\
\hline Perceived barriers for PA of the partner & $-85.4(26.3)$ & $-137.2--33.6$ & -0.12 \\
\hline Sport competence importance of the partner & $686.5(228.2)$ & $237.1-1135.9$ & 0.12 \\
\hline \multirow{2}{*}{$\begin{array}{l}\text { Perception of the partner of best friend social support } \\
\text { Sitting time }\end{array}$} & $213.9(50.7)$ & $114.1-313.7$ & 0.16 \\
\hline & & & \\
\hline Intercept & $9.72(0.2)$ & $9.4-10.1$ & \\
\hline PSPP-CY sport competence of the partner & $-0.61(0.2)$ & $-1.1--0.1$ & -0.12 \\
\hline
\end{tabular}

PSPP-CY: Physical Self-Perception Profile for Children and Youth

Note: Only the parameters for significant predictors were estimated 
1

2

3

4

5

6

7

8

9

10

11

12

13

14

15

16

17

18

19

20

21

22

23

24

25

26

27

28

29

30

31

32

33

34

35

36

37

38

39

40

41

42

43

44

45

46

47

48

49

50

51

52

53

54

55

56

57

58

59

60

Table 3: Specification of parameters (fixed effects) in final model [sitting time (h/day) as dependent variable] for non-reciprocal best friend dyads with predictors, with standard errors (SE), confidence intervals (Cl), and effects size (ES).

\begin{tabular}{llll}
\hline Parameter & Estimate (SE) & $95 \% \mathrm{Cl}$ & ES \\
\hline Intercept & $10.1(0.2)$ & $9.7-10.5$ & \\
$\begin{array}{l}\text { Sex of the actor (male }=1 \text {; female }= \\
\text { 0) }\end{array}$ & & $-1.1(0.3)-0.1$ & 0.10 \\
Sport competence importance of the & $-0.4(0.2)$ & $-.0 .8--0.02$ & -0.08 \\
$\begin{array}{l}\text { partner } \\
\text { Physical condition competence }\end{array}$ & $-0.4(0.2)$ & $-0.8--0.03$ & -0.08 \\
importance of the actor & & & \\
\hline
\end{tabular}

Note: Only the parameters for significant predictors were estimated 


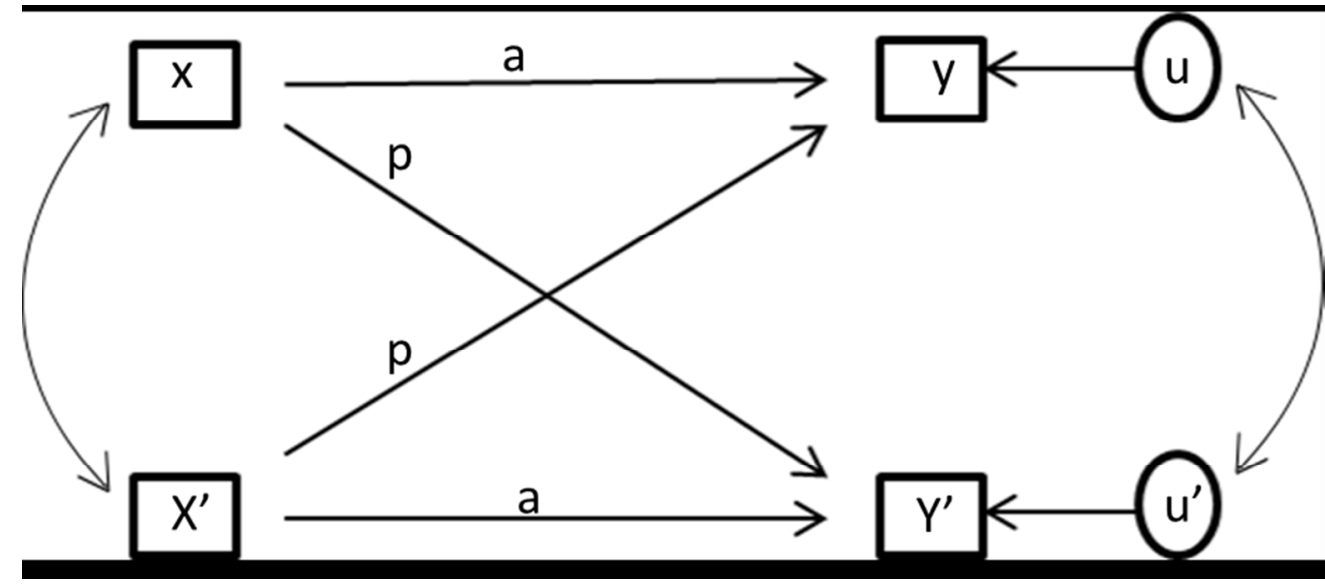

Actor-Partner Interdependence Model (APIM). Horizontal lines are actor effects (a) and diagonal lines are partner effects ( $p)$. Adapted from Kenny (1996) $157 \times 69 \mathrm{~mm}(150 \times 150 \mathrm{DPI})$ 